\title{
Relation entre le pH des sols et leur niveau de réceptivité à Fusarium solani var coeruleum et Fusarium roseum var sambucinum agents de la pourriture sèche des tubercules de pomme de terre
}

\author{
B Tivoli *, R Corbière, E Lemarchand \\ INRA, station de pathologie végétale, BP 29, 35650 Le Rheu, France
}

(Reçu le 29 mai 1989 ; accepté le 11 octobre 1989)

\begin{abstract}
Résumé - Une étude des relations entre le $\mathrm{pH}$ des sols et leur niveau de réceptivité à Fusarium roseum var sambucinum et à $F$ solani var coeruleum agents de la pourriture sèche des tubercules de pomme de terre est réalisée.

Dans le cas de 100 sols de culture dont les pH s'échelonnent de 5,1 à 6,2, un lien direct existe entre les faibles valeurs de $\mathrm{pH}$ et les faibles niveaux de réceptivité des sols à $F$ solani var coeruleum. Le point critique, entre sols réceptifs et sols qui ne le sont pas, se situe aux environs du $\mathrm{pH} 5,3$; en dessous de ce $\mathrm{pH}$, la majorité des sols sont résistants. Chez $F$ roseum var sambucinum, la distribution des sols non réceptifs est indépendante du $\mathrm{pH}$ : à chaque valeur de $\mathrm{pH}, 10$ à $20 \%$ des sols sont résistants. La confirmation expérimentale à partir d'un sol de lande de $\mathrm{pH}$ initial 3,9 amendé par de la chaux vive afin d'augmenter progressivement le $\mathrm{pH}$ corrobore les observations précédentes.

Dans le cas de $F$ solani var coeruleum, le parasite survit dans les sols réceptifs sous forme de chlamydospores, tandis que dans les sols non réceptifs, l'inoculum est entièrement lysé.

La différence de comportement de $F$ roseum var sambucinum et $F$ solani var coeruleum dans un même type de sol suggère des mécanismes de résistance des sols spécifiques à chacun des deux parasites.
\end{abstract}

écologie / lyse / chlamydospore / sol résistant / potentiel infectieux / chaulage

Summary - Relations between the $\mathrm{pH}$ of soils and their level of conduciveness to Fusarium solani var coeruleum and Fusarium roseum var sambucinum responsible for the dry rot of potato tubers. $A$ study of the relations between the $\mathrm{pH}$ of soils and their level of conduciveness to $\mathrm{F}$ solani var coeruleum and $\mathrm{F}$ roseum var sambucinum has been carried out.

In the 100 potato soils with $\mathrm{pH}$ values from 5.1 to 6.2 (fig 1), a direct relation is observed between the low values of $\mathrm{pH}$ and the suppressiveness of soils to $\mathrm{F}$ solani var coeruleum. The critical value between conducive and suppressive soils is about $\mathrm{pH}$ 5.3; under this $\mathrm{pH}$, most soils are suppressive. With $\mathrm{F}$ roseum var sambucinum, the frequency of suppressive soils is independent of the $\mathrm{pH}$; at each $\mathrm{pH}$ value, 10 to $20 \%$ of soils are suppressive. The experimental confirmation from a heathland soil with an initial $\mathrm{pH} 3.9$ which was subsequently limed to increase $\mathrm{pH}$, is in agreement with previous observations (fig 2, fig 3).

In the case of $\mathrm{F}$ solani var coeruleum, the pathogen persists in the conducive soils with chlamydospores whereas in the suppressive soils, the pathogen is completely lysed and the formation of resting spores is inhibited (fig 4).

ecology / lyse / chlamydospore / suppressive soil / inoculum potential / liming

\section{INTRODUCTION}

Les sols de culture de pomme de terre présentent différents niveaux de réceptivité aux pourritures sèches du tubercule de pomme de terre dues à $F$ solani var coeruleum et $F$ roseum var sambucinum (Tivoli et al, 1987). Un des sols étudiés a particulièrement retenu notre attention : il s'agit d'un sol de lande caractérisé par un $\mathrm{pH}$ extrêmement bas, de l'ordre de 4,0. Ce sol est à la fois très réceptif à $F$ roseum var sambucinum et inversement très peu réceptif à $F$ solani var coeruleum.

\footnotetext{
* Correspondance et tirés à part
} 
Cette observation nous a conduits à étudier plus précisément le rôle du $\mathrm{pH}$ en tant que facteur pouvant conditionner le niveau de réceptivité des sols à ces 2 agents pathogènes.

Les données bibliographiques concernant l'incidence du $\mathrm{pH}$ sur ces parasites sont, à notre connaissance, quasi inexistantes. Seul Small (1944) réisolant $F$ solani var coeruleum dans des sols de $\mathrm{pH}$ différents démontre que des valeurs de $\mathrm{pH}$ comprises entre 5,2 et 6,7 (c'est-à-dire aux seuls $\mathrm{pH}$ éprouvés par l'auteur) permettent la conservation de ce champignon.

L'incidence du $\mathrm{pH}$ sur les parasites fongiques telluriques et sur les Fusarium en particulier a été appréciée soit :

- en constatant de manière globale que la densité des populations de $F$ roseum dans le sol n'est pas modifiée pour des valeurs de $\mathrm{pH}$ comprises entre 4,9 et 7,3 (Kaufman et Williams, 1965);

- en étudiant l'effet du pH sur l'induction ou la modification des structures fongiques en condition de culture pure. Ainsi, Cappellini et Peterson (1969) montrent que la sporulation de Gibberella zeae s'effectue entre 5 et 9 avec un optimum à $\mathrm{pH} 8$ et Griffin (1976) précise qu'un milieu à $\mathrm{pH}$ 4,0 induit une plus grande formation de chlamydospores de $F$ solaniqu'à $\mathrm{pH} 6,0$;

- en tentant d'apprécier le rôle indirect du pH qui, par le biais de la modification de certains facteurs biotiques et/ou abiotiques, exerce une action "secondaire» sur les agents pathogènes.

En ce qui concerne les facteurs biotiques, les relations entre le $\mathrm{pH}$ du sol et certains groupes compétiteurs sont très étudiées : Marshall et Alexander (1960) montrent que l'activité antagoniste de Agrobacterium radiobacter sur F oxysporum f $\mathrm{sp}$ cubense est plus importante dans des sols acides $(5,7)$ ou neutres $(7,5)$. Entre ces valeurs de $\mathrm{pH}$, ce sont des facteurs tels que la texture du sol, son taux de matière organique et l'azote total, qui jouent le rôle le plus important. Dans le cas de Rhizoctonia solani, Chet et Baker (1980) précisent que les activités enzymatiques $\beta$ 1-3 glucanase et chitinase des Trichoderma harzianum présents dans les sols acides résistants à ce parasite sont actives à des $\mathrm{pH}$ respectifs de 4,5 et 5,3 .

En ce qui concerne les facteurs abiotiques, l'exemple le plus intéressant est celui de Scher et Baker (1982) relatif au $F$ oxysporum $f \mathrm{sp}$ lini. L'absence de fer libre serait responsable de la résistance de certains sols dans la mesure où cet élément est indispensable à l'élongation du tube germinatif des microconidies. Plus le sol est alcalin, moins le fer est disponible : les sols à pH 8 sont résistants ; à pH 6, le sol est sensible. Cette observation rejoint celle de Sneh et al (1984) avec le modèle $F$ oxysporum $\mathrm{f} \mathrm{sp}$ cucumerinum et les bactéries fluorescentes.

Ce travail se décompose en 2 parties :

- dans la première, à l'aide d'une enquête sur 100 sols de culture nous avons étudié la corrélation existant entre le $\mathrm{pH}$ des sols et leurs niveaux de réceptivité ;

- dans la seconde, l'incidence de la modification du $\mathrm{pH}$ du sol de lande sur les niveaux de réceptivité et sur l'évolution de l'inoculum est appréciée.

\section{MATÉRIEL ET MÉTHODES}

\section{Les terres}

Deux types de sols sont utilisés :

- les échantillons de sols de culture, au nombre de 100 , sont prélevés 1 mois après la récolte dans les parcelles ayant porté une culture de pomme de terre afin d'apprécier leur niveau de réceptivité.

Toutes ces terres sont séchées et tamisées comme il est indiqué par Tivoli et al (1987). Le pH-eau de ces terres est apprécié juste avant leur infestation ( $30 \mathrm{~g}$ de sol séché pour $75 \mathrm{ml}$ d'eau distillée). Les $\mathrm{pH}$ s'échelonnent de 5 à 6,2 et le nombre de sols dans chaque classe de $\mathrm{pH}$ est répertorié (tableau I) ;

- le sol de lande non cultivé de la région de Rennes (Saint-Thurial), de texture sableuse et de $\mathrm{pH} 3,9$.

Pour certaines expérimentations, le $\mathrm{pH}$ de ce sol est modifié artificiellement par incorporation au sol humidifié de chaux vive (à $80 \%$ de $\mathrm{CaO}$ ) à des doses variant de 0,2 à $1,5 \%$ (du poids du sol). Deux mois plus tard, les valeurs de $\mathrm{pH}$ sont stabilisées à $4,2,5,0$, $5,7,6,3,7,2$ et 7,8 .

\section{Les souches}

Les infestations artificielles des terres ainsi que les observations sur membranes de nitrate de cellulose sont réalisées avec deux parasites différents isolés à partir de tubercules de pomme de terre en voie de pourriture :

- F solani var coeruleum (souche B 33 collection Le Rheu),

- F roseum var sambucinum (souche A 44 collection Le Rheu).

Par commodité, ces parasites seront respectivement dénommés dans la suite du texte $F$ solani et Froseum.

\section{Appréciation de la réceptivité des sols}

Les sols sont infestés selon la méthode décrite précédemment (Tivoli et al, 1987) par une seule dose de macroconidies de chaque parasite : $16.10^{4} \mathrm{spores} / \mathrm{ml}$ de $F$ solani et $16.10^{5}$ spores $/ \mathrm{ml}$ de $F$ roseum. Les macroconidies sont obtenues par grattage superficiel d'une colonie fongique après incubation à $18^{\circ} \mathrm{C}$ pen- 
Tableau I. Répartition des 100 sols de culture en fonction du pH.

\begin{tabular}{|c|c|c|c|c|c|c|c|c|c|c|c|c|c|}
\hline $\mathrm{pH}$ & 5,0 & 5,1 & 5,2 & 5,3 & 5,4 & 5,5 & 5,6 & 5,7 & 5,8 & 5,9 & 6,0 & 6,1 & 6,2 \\
\hline Nombre de sols & 3 & 5 & 10 & 10 & 10 & 11 & 6 & 10 & 8 & 9 & 8 & 4 & 6 \\
\hline
\end{tabular}

dant 2 semaines sur un milieu de culture gélosé à base d'extrait de malt à $2 \%$. Après incubation à $15^{\circ} \mathrm{C}$ pendant 4 semaines, les sols sont étalés sur des tranches de demi-tubercules fraîchement sectionnés. Les notations réalisées après 2 semaines d'incubation à $15^{\circ} \mathrm{C}$ permettent de rendre compte des niveaux de potentiel infectieux des sols exprimés par les indices de gravité (IG); ceux-ci varient de 0 pour un potentiel infectieux nul à 100 pour un potentiel infectieux maximal.

\section{Observations de l'inoculum}

Les macroconidies sont déposées sur les membranes de nitrate de cellulose lors d'une filtration rapide sous vide de $5 \mathrm{ml}$ de suspension à $5.10^{5} \mathrm{spores} / \mathrm{ml}$ (Tivoli et al, 1983). Ces membranes sont enfouies entre 2 couches de sol humidifié dans des boîtes de Petri; puis l'ensemble est mis à incuber à $23^{\circ} \mathrm{C}$. L'évolution de l'inoculum est appréciée par observation microscopique en considérant 3 critères : la germination des spores, la lyse de l'inoculum et la formation de nouvelles structures (chlamydospores ou nouvelles conidies). Les pourcentages sont déterminés à partir du comptage de 50 conidies par lame et de 3 lames par traitement.

\section{RÉSULTATS}

\section{Relation entre le $\mathrm{pH}$ des sols et leur niveau de réceptivité}

Les niveaux de potentiel infectieux des sols infestés sont variables : certains, faibles $(I G<40)$, caractérisent des sols peu réceptifs; d'autres, beaucoup plus élevés (IG>70), indiquent des sols très réceptifs. Nous avons calculé, pour chaque agent pathogène, un IG moyen pour tous les sols présentant une même valeur de $\mathrm{pH}$ (fig 1). Cette figure indique clairement que la relation $\mathrm{pH}$-réceptivité est différente pour $F$ solani et $F$ roseum :

- en ce qui concerne $F$ roseum, la distribution des sols non réceptifs est indépendante du $\mathrm{pH}$ : pour chaque valeur de $\mathrm{pH}, 10$ à $20 \%$ des sols sont résistants (fig 1b). Cette situation entraîne, pour chaque valeur de $\mathrm{pH}$, une moyenne des IG relativement constante qui se situe entre 50 et 60 (fig 1a) ;

- chez F solani (fig 1a), une très bonne corrélation existe entre les IG et les valeurs de $\mathrm{pH}$. Le
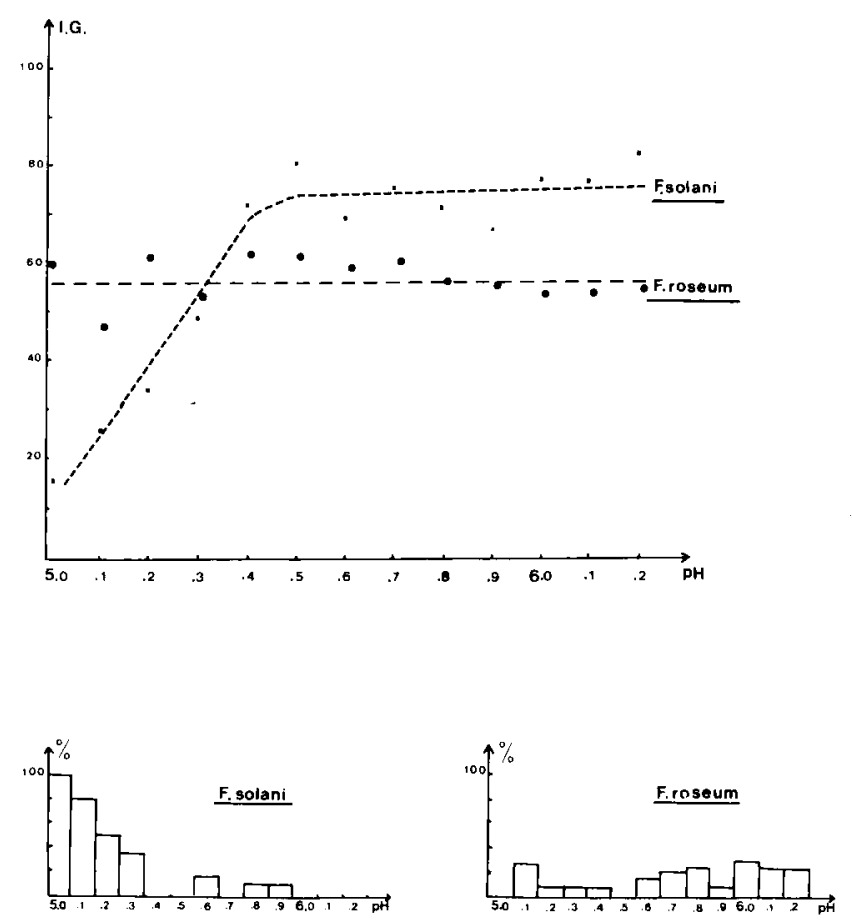

Fig 1. Relation entre le $\mathrm{pH}$ de 100 sols de culture et leurs niveaux de réceptivité à $F$ solani ou $F$ roseum exprimés par : a : la moyennes des indices de gravité (IG) à chaque valeur de $\mathrm{pH} ; \mathbf{b}$ : la fréquence des sols non réceptifs à chaque valeur de $\mathrm{pH}$.

nuage de points obtenu s'ajuste avec une précision correcte $\left(r^{2}=0,72\right)$ à une courbe dont l'équation est un polynôme du $3^{\theta}$ degré. Le $\mathrm{pH}$ 5,3 constitue une limite entre les sols non réceptifs $(\mathrm{pH}<5,3)$ et les sols très réceptifs $(\mathrm{pH}>5,3)$. Ainsi, la fréquence des sols non réceptifs à pH 5 , $5,1,5,2$ et 5,3 est respectivement de $100,80,50$ et $30 \%$ pour atteindre des valeurs proches de 0 à des $\mathrm{pH}$ supérieurs (fig $1 \mathrm{~b}$ ).

\section{Confirmation expérimentale : niveau de réceptivité d'un sol de lande dont le pH est modifié}

Les résultats obtenus à partir du sol de lande dont le $\mathrm{pH}$ est artificiellement modifié (fig 2), confirment les observations précédentes :

- chez $F$ roseum, les variations des valeurs de $\mathrm{pH}$ n'entraînent pas de modifications importantes des IG : ceux-ci, toujours supérieurs à 60 , rendent compte de sols très réceptifs ; 


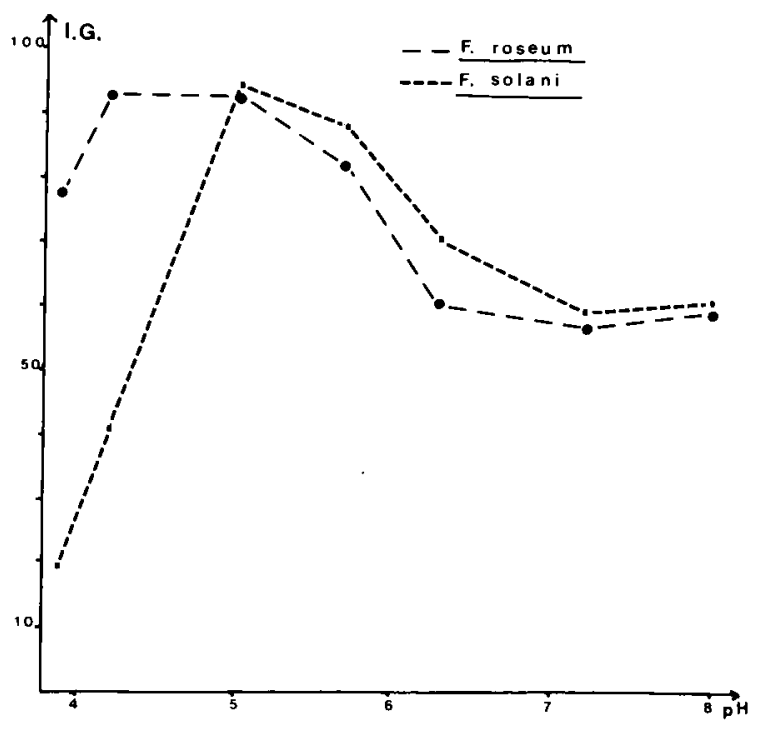

Fig 2. Relation entre les niveaux de $\mathrm{pH}$ d'un sol de lande amendé en chaux et les niveaux de réceptivité à $F$ solani ou $F$ roseum exprimés par les indices de gravité (IG).

- chez $F$ solani, I'IG, très faible au pH 4 initial (IG $=18,8$ à pH 3,9), augmente rapidement jusqu'au $\mathrm{pH} 5$ pour atteindre des valeurs supérieures à 90 pour des $\mathrm{pH}$ supérieurs, à $5^{\circ} \mathrm{C}$. Enfin, chez $F$ solani, si l'influence des faibles niveaux de $\mathrm{pH}$ se confirme, on peut cependant observer un certain décalage du $\mathrm{pH}$ "charnière" par rapport aux sols naturels $(5,3$ chez ces derniers, inférieur à 5 chez le sol de lande amendé).

\section{Évolution de l'inoculum au contact d'un sol de lande dont le $\mathrm{pH}$ est modifié}

L'inoculum, supporté par des membranes filtrantes, est placé au contact d'une terre de lande dont le pH initial $(3,9)$ est modifié par incorporation de chaux vive, jusqu'à des $\mathrm{pH}$ de 5,9, 7,0 et 8,6 . L'évolution de l'inoculum est suivie pendant une période de 3 mois. II est à noter qu'au terme de cette expérience, le pH des sols amendés a diminué de 0,5 unité.

L'évolution des pourcentages des différents types de propagules et de leur lyse est représentée sur la figure 3. Le pouvoir germinatif des conidies et l'élongation des filaments germinatifs sont très difficiles à estimer car une lyse importante intervient dès les premiers jours.

Chez $F$ roseum, la lyse varie peu d'un $\mathrm{pH}$ à l'autre ; elle a toutefois tendance à être plus rapide aux $\mathrm{pH}$ les plus bas : par exemple, pendant les premiers jours de contact entre l'inoculum et le sol, $50 \%$ des filaments sont lysés à $\mathrm{pH}=3,9$, tandis que seulement $30 \%$ d'entre eux le sont aux autres $\mathrm{pH}$. Cette différence s'estompe rapidement : la lyse augmente progressivement avec le temps et devient importante après 3 mois d'incubation à tous les $\mathrm{pH}$.

Chez $F$ solani, le $\mathrm{pH}=3,9$ se distingue nettement des autres. Aux pH supérieurs à 3,9 , la lyse débute très tôt puisque $80 \%$ des cellules conidiennes et des filaments ont disparu en $7 \mathrm{j}$; à $\mathrm{pH}=3,9$, elle progresse, au contraire, plus lente-
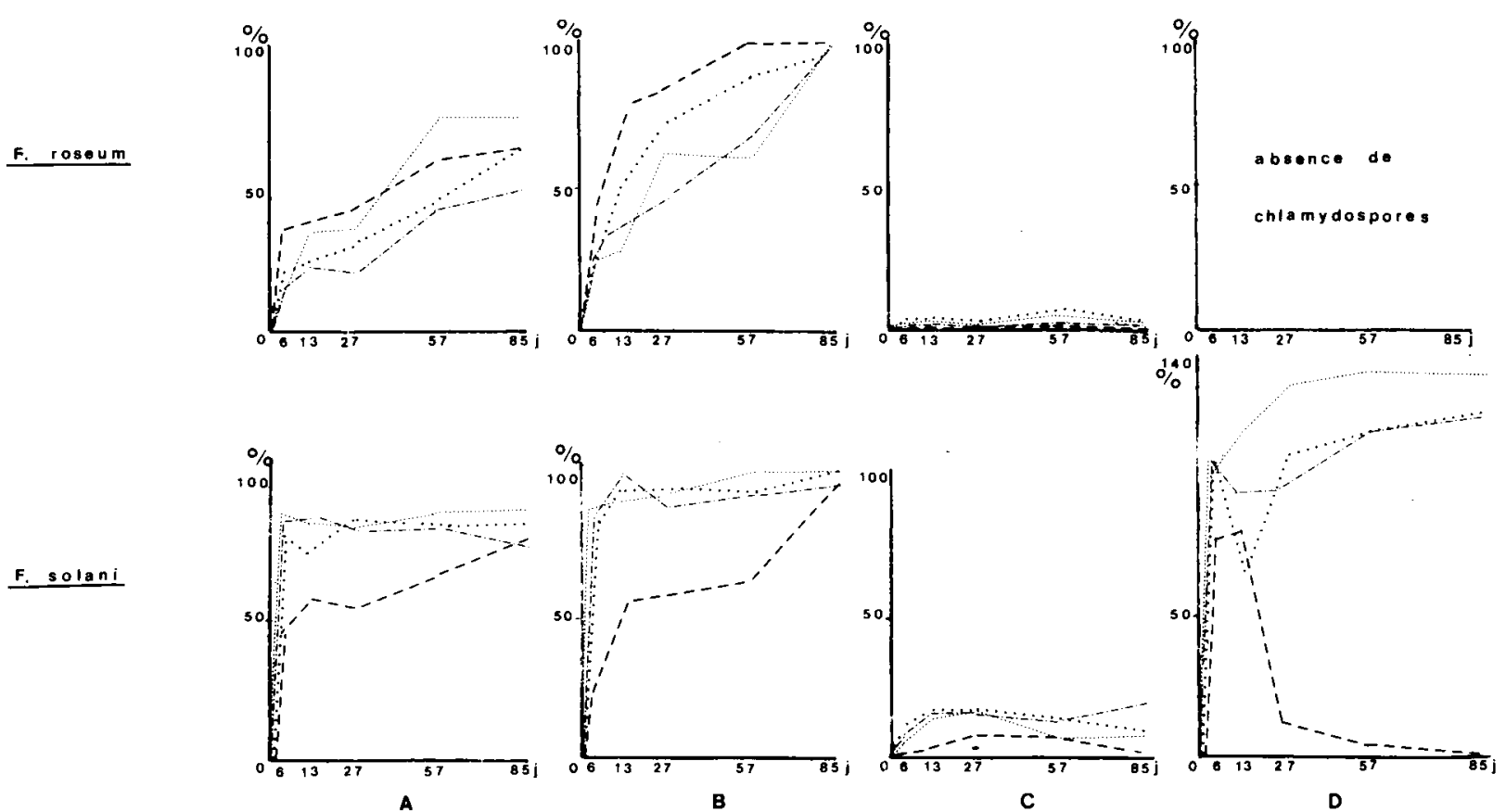

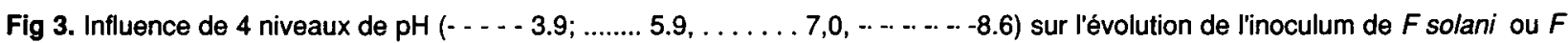
roseum exprimée par : $\mathrm{A}=\%$ de cellules conidiennes vides ; $\mathrm{B}=\%$ de filaments germinatifs lysés ; $\mathrm{C}=\%$ de cellules conidiennes transformées en chlamydospores; $D=$ nombre de chlamydospores sur les filaments (pour cent conidies). 
ment : elle atteint $45 \%$ des cellules et $30 \%$ des filaments. Cette différence se maintient pendant 2 mois; cependant, quel que soit le $\mathrm{pH}, 100 \%$ des filaments et $80 \%$ des cellules sont lysés après 3 mois de conservation dans le sol.

Le nombre de chlamydospores formées à partir des conidies de $F$ roseum est faible, à toutes les valeurs de $\mathrm{pH}$, mais quelques cellules à paroi épaissie apparaissent. L'influence du pH est plus nette sur la formation et la conservation des chlamydospores de $F$ solani. A partir des conidies, à $\mathrm{pH}=3,9$ leur nombre est beaucoup plus réduit qu'aux autres $\mathrm{pH}$. A partir des filaments, à ce même $\mathrm{pH}$, elles apparaissent dès les premiers jours sous forme de petites chlamydospores atypiques qui sont ultérieurement lysées après 2 semaines d'incubation. Aux $\mathrm{pH}$ supérieurs, elles sont plus volumineuses, leur nombre augmente régulièrement pendant les 3 mois (fig 4) permettant ainsi à l'inoculum de se conserver dans le sol.

Les variations de $\mathrm{pH}$ sont à l'origine de modifications importantes du comportement de $F$ solani. Elles affectent à la fois la lyse et la formation ou la conservation des chlamydospores; chez $F$ roseum, il se produit une lyse progressive au cours du temps, avec une formation très réduite de "chlamydospores" dans les cellules conidiennes.

\section{CONCLUSION}

L'ensemble de ce travail amène plusieurs commentaires.

Par rapport au travail précédent sur la réceptivité de différents sols aux fusarioses de la pomme de terre (Tivoli et al, 1987), cette étude tend à démontrer que les mécanismes de résistance des sols ne sont pas identiques pour les 2 maladies étudiées. Dans le cas de $F$ solani, le lien direct entre les faibles niveaux de réceptivité et les faibles $\mathrm{pH}$ est démontré (que ce soit à partir de sols "naturels» ou d'un sol dont les niveaux de $\mathrm{pH}$ ont été artificiellement modifiés). Le point critique entre sols réceptifs et sols qui ne le sont pas se situe aux environs du $\mathrm{pH}$ 5,3. Cette relation entre le $\mathrm{pH}$ et le niveau de réceptivité des sols n'est pas unique : dans le cas du Plasmodiophora brassicae (Rouxel et Briard, 1988; Doublet et al, 1988), la corrélation inverse entre ces 2 facteurs est démontrée.

II apparaît, par ailleurs, que la différence entre sols réceptifs ou non à $F$ solani repose sur une évolution distincte de l'inoculum : la phase de germination, apparemment identique dans les 2 types de sols, se poursuit dans les sols réceptifs par une lyse des cellules conidiennes et mycéliennes beaucoup plus rapide que dans les sols non réceptifs, et par une formation considérable de chlamydospores. Dans les sols non réceptifs, l'absence de chlamydospores conduit à une disparition de l'inoculum. Cette observation rejoint celle de Burke (1965) qui compare des sols sensibles et résistants à $F$ solani f sp phaseoli : comme dans notre exemple, la lyse du mycélium et des tubes germinatifs a lieu dans les 2 sols, de sorte que l'organisme survit seulement sous forme de chlamydospores qui sont de plus grande taille et plus nombreuses dans les sols sensibles.

Enfin, en ce qui concerne $F$ roseum, les différentes observations le montrent, aucune relation n'existe entre le $\mathrm{pH}$ et le niveau de réceptivité des sols. Les expériences réalisées avec le sol

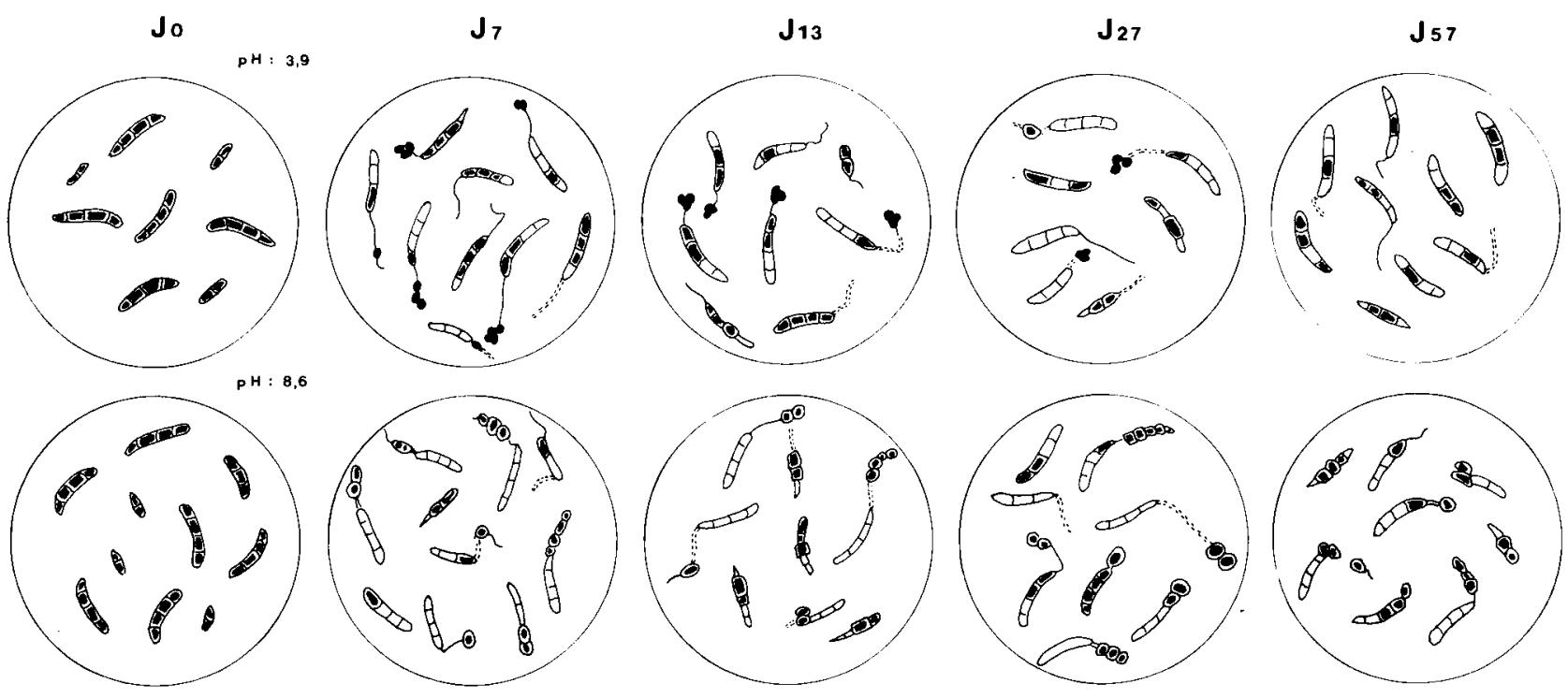

Fig 4. Évolution de l'inoculum de $F$ solani au bout de $7,13,27$ et $57 \mathrm{j}$ à $23^{\circ} \mathrm{C}$ dans le sol de lande initial $(\mathrm{pH} 3,9)$ ou amendé en chaux (pH 8,6). 
de lande, sol très réceptif à ce parasite, dans lequel les valeurs de $\mathrm{pH}$ sont artificiellement augmentées, montrent que le niveau de réceptivité n'est que très peu modifié, et que l'évolution de l'inoculum est identique à celle qui a lieu dans le sol initial. Ce sol conserve donc ses propriétés biologiques à l'égard de ce parasite, indépendamment du $\mathrm{pH}$.

Les mécanismes susceptibles de régir les faibles niveaux de réceptivité des sols acides visà-vis de $F$ solani var coeruleum sont en cours d'étude. Deux types de facteurs distincts mais sans doute complémentaires sont à approfondir : - des facteurs abiotiques agissant directement ou non par le biais du $\mathrm{pH}$,

- des facteurs biotiques intervenant au niveau de la compétition entre le parasite et la microflore tellurique.

\section{RÉFÉRENCES}

Burke DW (1965) Fusarium root rot of beans and behaviour of the pathogen in different soils. Phytopathology, 55, 1 122-1 126

Cappellini RA, Peterson JL (1969) Sporulation of Gibberella zeae. II - The effects of pH on macroconidium production. Mycologia, 61, 481-485

Chet I, Baker R (1980) Induction of suppressiveness to Rhizoctonia solani in soil. Phytopathology, 70, 994998

Doublet L, Rouxel F, Masson JP (1988) Réceptivité des sols à la Hernie des Crucifères : analyse statistique des résultats. Agronomie, 8, 459-464

Griffin GJ (1976) Roles of low pH, carbon and inorga- nic nitrogen use in chlamydospore formation by Fusarium solani. Can J Microbiol, 22, 1 381-1 389

Kaufman DD, Williams LE (1965) Influence of soil reaction and mineral fertilization on numbers and types of fungi antagonistic to four soil-borne plant pathogens. Phytopathology, 55, 570-574

Marshall KC, Alexander M (1960) Competition between soil bacteria and Fusarium. Plant Soil, 12, 143-153

Rouxel F, Briard M (1988) Studies of soil receptiveness to clubroot caused by Plasmodiophora brassicae: experiments on responses of a series of vegetable soils in Brittany. Proc CEC/IOBC Experts'Group Meeting, Rennes, 20-22 nov 1985

Scher FM, Baker R (1982) Effect of Pseudomonas putida and a synthetic iron chelator on induction of soil suppressiveness to Fusarium wilt pathogens. Phytopathology, 72, 1 567-1573

Simeoni LA, Lindsay WL, Baker R (1987) Critical iron level associated with biological control of Fusarium wilt. Phytopathology, 77, 1 057-1 061

Small T (1944) Dry rot of potato (Fusarium coeruleum (Lib) Sacc). Investigation on the sources and time of infection. Ann Appl Biol, 31, 290-295

Sneh B, Dupler M, Elad Y, Baker R (1984) Chlamydospore germination of Fusarium oxysporum $f \mathrm{sp}$ cucumerum as affected by fluorescent and lytic bacteria from Fusarium-suppressive soil. Phytopathology 74, 1 115-1 124

Tivoli B, Corbière R, Jouan B (1983) Influence de la température et de l'humidité sur le comportement dans le sol de 3 espèces ou variétés de Fusarium responsables de la pourriture sèche des tubercules de pomme de terre. Agronomie 3, $1001-1009$

Tivoli B, Tika N, Lemarchand E (1987) Comparaison de la réceptivité des sols aux agents de la pourriture des tubercules de pomme de terre: Fusarium spp et Phoma sp. Agronomie, 7, 531-538 